Twenty-Seventh Symposium (Intemational) on Combustion/The Combustion Institute. 1998/pp. 537-544

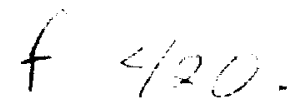

\title{
TWO-DIMENSIONAL FAILURE WAVES AND IGNITION FRONTS IN PREMIXED COMBUSTION
}

\author{
T. G. VEDARAJAN, ' J. BUCKMASTER,' AND P. RONNEY, \\ 'University of Illinois \\ Department of Aeronautical o Astronautical Engineering \\ Urbana, IL 61801, L'SA \\ 'University of Southem California \\ Department of Mechenical Engineering \\ Los Angeles, CA 90089 , USA
}

\begin{abstract}
This paper is a continuation of our work on edge-flames in premixed combustion. An edge-flame is a two-dimensional structure constructed from a one-dimensional configuration that has two stable solutions (bistable equilibrium). Edge-flames can displav wavelike behavior, advancing as ignition fronts or retreating as failure waves. Here we consider two one-dimensional configurations: twin deflagrations in a straining Alow generated by the counterflow of fresh streams of mixture: and a single deflagration subject to radiation losses. The edge-flames constructed from the first configuration have positive or negative speeds, according to the value of the strain rate. But our numerical solutions strongly suggest that only positive speeds (corresponding to ignition fronts) can exist for the second configuration. We show that this phenomenon con also occur in diffusion flames when the Lewis numbers are small. And we discuss the asymptotics of the one-dimensional twin deflagration configuration. an overiooked problem from the $70 \mathrm{~s}$.
\end{abstract}

\section{Introduction}

tis

In edge-lame can be defined, roughly speaking in flame sheet with an edge. There is a growing inture on edge-tlames in non-premixed combus1. (e.g., Ref. [I]) reflecting their important role. A ine spreading over a fuel bed, solid or liquid, will Twe an edge; edge flames are an important part of 4. structure of the combustion field that occurs in 1.4 burning of heterogeneous solid propellants [2]; * fivy are a noticeable characteristic of candle flames or microgravity conditions [3]; and they must ex2 Thenever a hole is torn in a flame-sheet by turfent eddies, so that their behavior is relevant to problem of lifted turbulent diffusion flames Important theoretical work has been piohened by Dold and coworkers $[6,7]$.

dige-flames in premixed combustion have been well studied. Indeed, there appears to be only a in theoretical treatment [8] and a single explicit Herimental study [9]. Old expenimental evidence their existence [10] is noted in Ref. [8]. The presPaper is a sequel to Ref. [8] and examines preedge-flames in two hitherto unexamined conin urations.

tomple framework in which edge-flames can be Aructed proceeds in the following fashion: There tortain one-dimensional combustion systems, jondent on a spatial variable $x$, say, for which are multiple solutions. Of particular interest Wistable systems for which there are three soluins, two stable and one unstable, the counterflow diffusion flame being a well-known example. Suppose the weakest (strongest) of the stable solutions has a temperature distribution $T_{1}(x)\left[T_{2}(x)\right] . T_{1}(x)$ will often correspond to a quenched state, or a close approximation thereof, with values close to some background or supply temperature $T_{f}$. The maximum value of $T_{2}(x)$ will be close to a flame-temperature (e.g., the adiabatic flame-temperature or the Burke-Schumann Aame-temperature). An unsteady two-dimensional combustion field can then be defined by an initial-value problem in the $x-z$ plane, where the boundary conditions in $x$ are those for the one-dimensional problem, and

$$
T(x, z, t) \rightarrow T_{2}(x), T_{1}(x) \text { as } z \rightarrow+\infty,-\infty
$$

If $T_{2}(x)$ is associated with a single thin reaction zone (flame-sheet) and $T_{1}(x)$ is associated with negligible reaction, an edge-flame is defined and the edgeflame structure either moves in the direction of decreasing $z$, in which case we call it an ignition front, or moves in the direction of increasing $z$, in which case we call it a failure wave. Here we continue the discussion of ignition fronts and failure waves in the premixed context. We discuss the situation where the one-dimensional problem is defined by a twinflame counterflow, and we also discuss a deflagration in which multiple solutions arise because of radiation losses. Some related results on diffusion flames are briefly discussed. as is the asymptotic description of quenching for the twin-flame problem. As the latter 


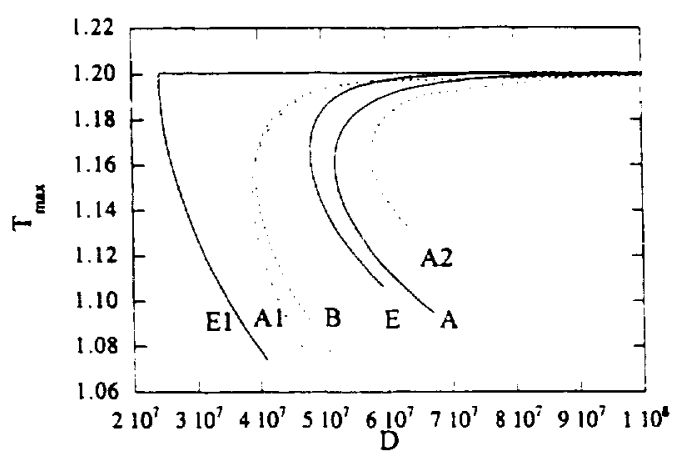

FIG. 1. Variations of maximum temperature with Damköhler number for twin counterflow flames: E, exact: E1, first-order approximation of exact solution: $A$, solution of the autonomous equation $A 10 ; A 1,(A 19), 1$ term in $q ; A 2$, (A19), 2 terms in $q ; B,(A 20)$.

will only be of interest to the asvmptotics community, it is relegated to the Appendix.

This work and Ref. [8] are parts of the dissertation of Vedarajan [11].

\section{The Twin-Flame Counterflow Problem}

Consider a symmetric counterflow of fresh mixture that, in general, supports twin flames. This configuration has long been studied, both theoretically and experimentally.

A simple model suitable for our purposes starts with the equation

$$
\begin{aligned}
& -\rho \alpha x d / d x\left(C_{p} T, Y\right)=\lambda C_{p}^{-1} d^{2} / d x^{2}\left(C_{p} T, Y\right) \\
& +B Y e^{-E / R T}(Q,-1)
\end{aligned}
$$

where $\alpha$ is the rate of strain and we have assumed that the Lewis number is 1 . The supply conditions are

$$
\underline{|x| \rightarrow \infty} T \rightarrow T_{f}, Y \rightarrow Y_{f}
$$

If $\Delta T_{a} \equiv Q Y_{f} / C_{p}$ is used as a reference temperature, and

$$
l_{c}=\sqrt{\lambda \rho C_{p} \alpha}
$$

is used as a reference length, a single nondimensional equation can be deduced, namely,

$$
\begin{gathered}
-x T_{x}=T_{x a}+D\left(1+T_{f}-T\right) e^{-a T}, \\
D=B / \rho \alpha, \theta=E / R \Delta T_{a}
\end{gathered}
$$

Because of the symmetry, it is sufficient to solve this equation in $x>0$ with the boundary conditions

$$
T_{x}(0)=0, T(\infty)=T_{f}
$$

Note that within a context that resolves the coldboundary difficulty (e.g., a cutoff temperature), the system of equations 4 and 5 has a stable quenched solution in which $T=T_{f}$ everywhere. In addition. it is well known that for $D$ greater than some minimum value $D_{\min }$, there are two solutions. These can be characterized by the maximum value of $T\left(T_{\max }\right)$, and a representative response, obtained numerically, is shown as a solid line in Fig. 1 (curve E). Here $\dot{T}_{f}=$ $0.2, T_{a} \equiv 1+T_{f}=1.2$, and $\theta=16$, values adopted throughout the paper. The upper branch corresponds to stable solutions, and it is these, along with the quenched solutions, that are the kev ingredients of the unsteady two-dimensional problem described in general terms in the Introduction. We are particularly concerned with solutions for values of $D$ close to $D_{\text {rnin }}$.

As an aside, not part of the main thrust of our discussion, we note that there have been asvmptotic treatments of the system of equations 2 and 3 in lieu of a numerical strategy, but there are ingredients that have not been discussed before. and we describe these in the Appendix.

\section{Failure Waves and Ignition Waves}

In this section, we use the strategy described in the Introduction to define a two-dimensional unsteady problem with $T_{2}(x)$ defined by a point on the upper branch of Fig. 1 (curve E) and $T_{1}(x) \equiv T_{f}$, the quenched state. The appropriate generalization of equation 4 is

$$
T_{t}-x T_{x}=T_{x x}+T_{z z}+D\left(T_{a}-T\right) e^{-w T}
$$

now accounting for diffusion in the $\approx$ direction as well as in the $x$ direction.

Note that as $|z| \rightarrow \infty$, where $T_{:=} \rightarrow 0$, equation 6 reduces to the one-dimensional form (equation 4 ), and the solution $T_{2}(x)$ can be assigned as $z \rightarrow+\infty$, the solution $T_{1}(x) \equiv T_{f}$ as $z \rightarrow-\infty$. (Note the remark following equation 5.) Boundary conditions in $x$ are those for the one-dimensional problem, namely, equation 5. And initial conditions, whose precise form is not important, are defined by a simple smooth interpolation between $T_{1}$ and $T_{2}$.

Figure 2a shows an initial temperature profile. Note that at $z=-5$, it is $T_{1}=T_{f}$, and at $z=+15$, it is $T_{2}(x)$, the one-dimensional twin-flame configuration. The Damköhler number is $4.9 \times 10^{i}$ and the Alame sheets are merged together, because we are close to $D_{\min }$. Figure $2 \mathrm{~b}$ shows the profile at a later time, and it is clear that the structure is retreating, corresponding to a failure wave.

In view of the double-flame structure, it might be argued that the use of the rubric "edge-flame" is not appropriate, but the behavior revealed here has its counterpart in the edge-flames discussed in Ref. [8], where the single-flame counterflow problem is examined (fresh versus inert counterflow).

If $\alpha$ is decreased ( $D$ increased), it is possible to 
le quenched $n$ addition. it me minimum These can be $T\left(T_{\text {mux }}\right)$, and umerically, is ). Here $T_{f}=$ lues adopted ranch corre. ie. along with $v$ ingredients in described IVe are parvalues of $D$

hrust of our $\mathrm{n}$ asymptotic and 3 in lieu ingredients and we de-

Vaves

described in ensional unpoint on the $(x) \equiv T_{f}$, the ralization of

$\Gamma l e^{-\theta T T}$

direction as

equation 6 equation 4), is $z \rightarrow+\infty$ - the remark lons in $x$ are ' $m$, namely, lose precise y a simple $\therefore$.

rure profile. it $z=+15$, ine configu$10^{7}$ and the luse we are ile at a later ; retreating,

it might be lame" is not here has its I in Ref. [s], blem is expossible to
PREMIXED EDGE FLAMES

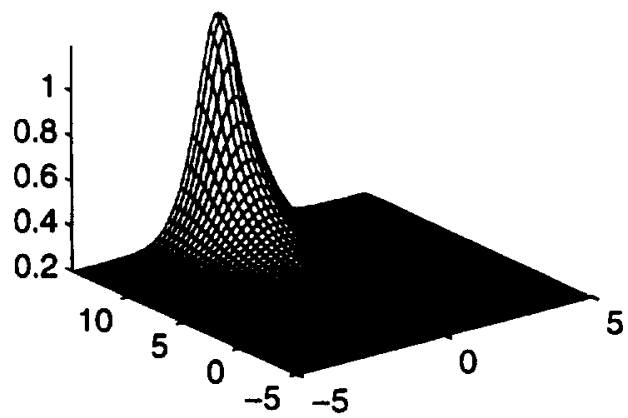

Fic. 2. Temperature profiles of a failure wave $D=4.9 \times 10^{-}:(a) t=0:(b) t=4.5$.

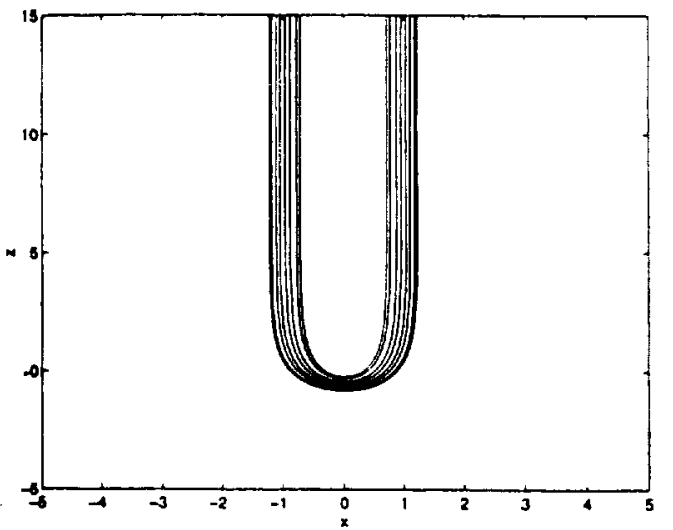

FIc. 3. Reaction-rate contours of an ignition front $D=$ $1.1 \times 10^{8}, t=4.5$. Values are $(0.5,1.0,3.0,5.0,5.0,3.0$, $1.0,0.5]$.

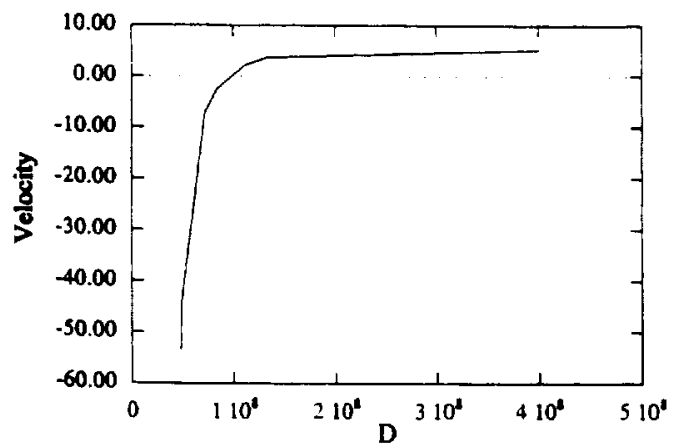

Fic. 4. Variations of wave speed (edge speed) with $\mathrm{Da}$ mkobler number, twin flames.

get an ignition front, and Fig. 3 shows reaction-rate contours in such a case. Steepening of the leading (lower edge) portion of the flame is quite apparent. Here (and also for the failure wave) the front is of unchanging shape following the passage of initial transients.
By performing a number of calculations for different values of $D$, it is possible to construct a graph showing variations of the front speed $V$ with $D$ (Fig. 4). Here

$$
V=(\dot{*}) . S^{-1}
$$

where $(\dot{*})_{+}$is the dimensional front speed and $S$ is the adiabatic flame speed defined by the system. The quenching value of $D\left(D_{\min }\right)$ is $4.802 \times 10^{\circ}$, and for values of $D$ between $D_{\min }$ and $D_{0}\left(9.776 \times 10^{7}\right)$, the front speed is negative. But for values of $D$ greater than $D_{10}$, the front speed is positive. The significance of results such as these, both in the present context and in the single-flame context of Ref. [8], is that flames can experience two distinct types of quenching: global quenching, arising when $D$ is reduced below the value $D_{\text {mini }}$, and quenching if part of the flame has been destroyed and $D_{\min }<D<D_{0}$ so that a failure wave enlarges the region of destruction.

Resuits such as these and the one-dimensional analog discussed in Ref. [8] suggest that the basic ingredients we have included here-multivaluedness, quenching-will always lead to the dichotomy of positive and negative speeds for the two-dimensional structure. That this is not the case is shown by the example discussed in the next section.

\section{Multivaluedness Due to Radiation Losses}

Consider the equation for a plane deflagration, radiation losses included.

$$
\begin{gathered}
M C_{p} T_{x}=\lambda T_{x x}+B Q Y e^{-E / R T}-q^{\prime \prime \prime} \\
q^{\prime \prime \prime}=10^{3} \overline{M W} P_{: t+m}\left(Y_{f}-Y^{\prime}\right)\left(T-T_{f}\right) W / m^{3}
\end{gathered}
$$

where $\overline{M W}$ is the mean molecular weight, $P_{\text {itun }}$ is the total pressure in atmospheres, and $T$ is the temperature in degrees Kelvin. We suppose that $Y$ represents methane and the radiating species are $\mathrm{CO}_{2}$ and $\mathrm{H}_{2} \mathrm{O}$, whose concentrations are proportional to $\left(Y_{f}-Y\right)$. There is a corresponding equation for $Y$ (cf. equation 


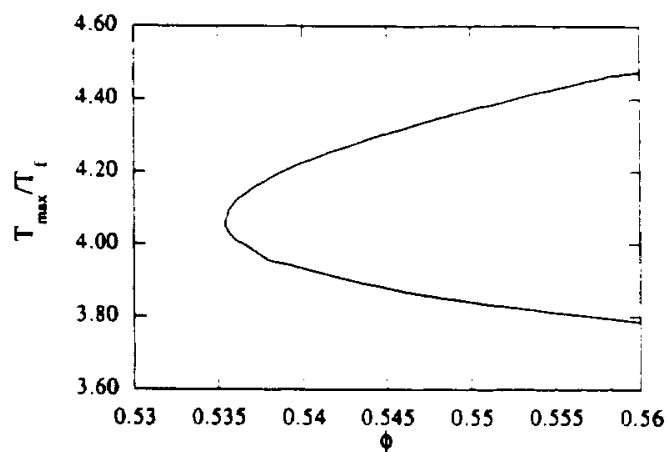

Fig. 5. Variations of flame temperature with equivalence ratio for a deflagration with radiation losses.

2). The expression for $q^{\prime \prime \prime}$ is derived bv first constructing an accurate representation of the temperature dependence of the band radiation for each species and then fitting this with a straight line, a surprisingly reasonable approximation up to a temperature of $1800 \mathrm{~K}$. All other parameters are chosen so that the model provides a reasonable approximation of methane/air flames in the neighborhood of the lean limit. The goals are only qualitative, but we do not wish to be led astray by gross quantitative inaccuracies.

Now the system of which equation 8 is a part defines, in addition to the quenched solution $T=T_{f}$, $Y=Y_{f}$, dual solutions for values of $Y_{f}$ greater than

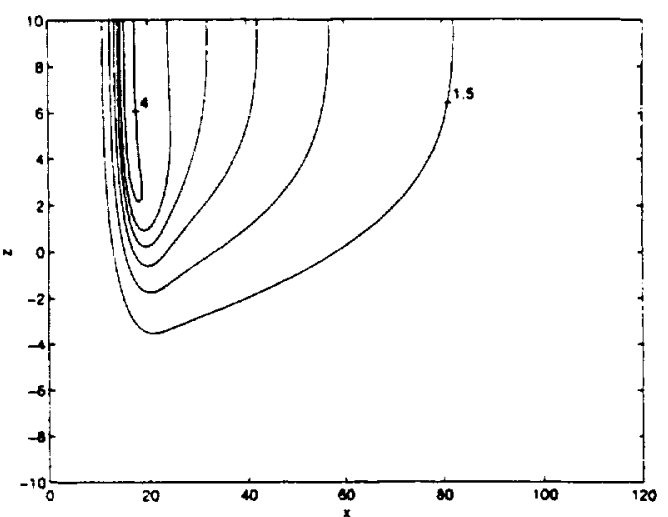

FIG. 7. Temperature contours for an ignition front with radiation losses, $\phi=0.53556$. Values are $(1.5,2.0,2.5,3.0$. $3.5,4.0$.

the radiation-defined inflammability limit $Y_{f}=$ 0.02945 (equivalence ratio $\phi=0.5355$ ): See Fig. 5 , the upper branch of which corresponds to stable solutions. Thus we can construct a two-dimensional unsteady combustion field (an edge-flame), and Fig. 6 shows an ignition wave for $\phi=0.53556$, a value close to the limit. Temperature and reaction-rate contours are shown in Figs. 7 and 8 . The wave is quite thick because the temperature decav occurs on a scale that is much larger than the preheatzone thickness. Figure $9 \mathrm{a}$ shows variations of the

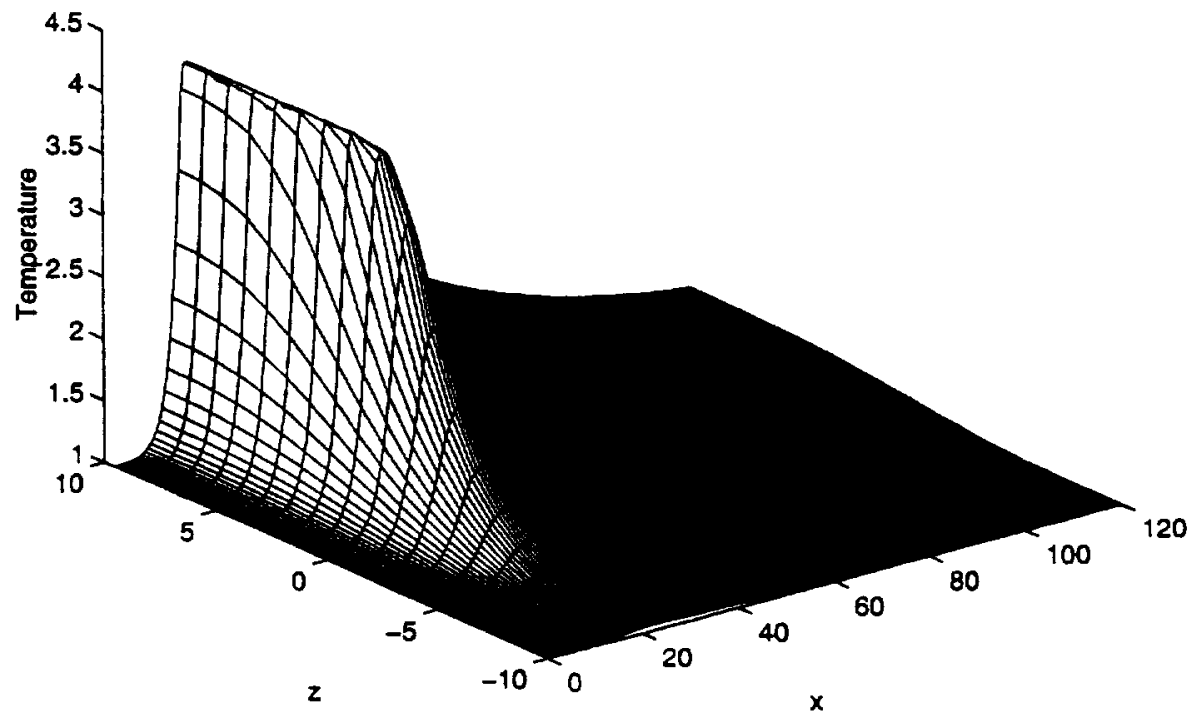

F1G. 6. An ignition front in a flame with radiation losses, $\phi=0.53556$. 


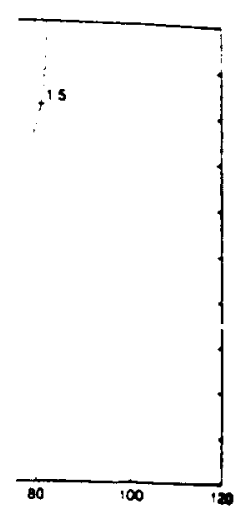

In ignition front with s.e 11.5. 2.0. 2.5. 3.0,

bility limit $r_{i}=$ 1.5355): See Fig. 5 . ponds to stable soa two-dimensional ge-tlamel, and Fig. $=0.53556$, a value and reaction-rate nd 8 . The wave is ure decav occurs on han the preheat$s$ variations of the

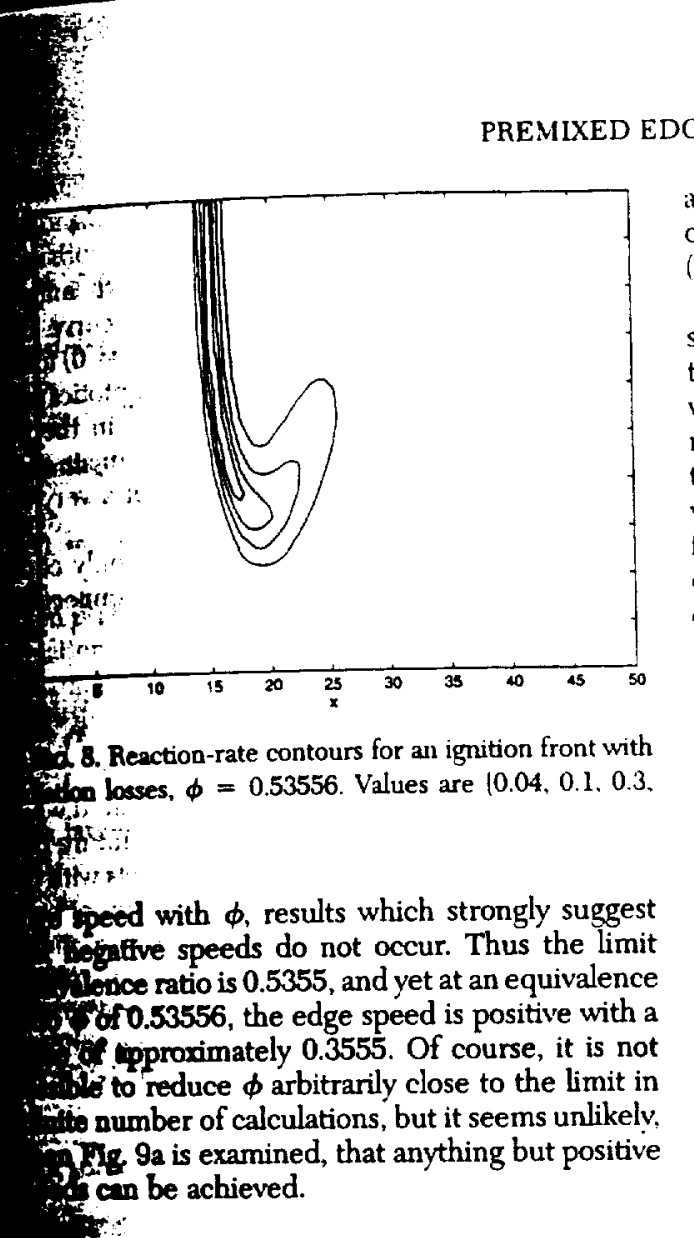

also a characteristic of existing results when the underlving one-dimensional flane is a diffusion flame (e.g., Ref. [1]).

On the other hand, if a hole is tom in a flame subject to radiation losses, the hole will heal no matter how close the equivalence ratio is to the limit value. It is natural to wonder if robust flames of this nature can occur in the counterflow configuration if the Lewis number is different from 1 , and although we have no results of this nature for premixed Hames, we have uncovered robust edges in the case of diffusion flames. Figure $9 \mathrm{~b}$ shows variations of edge speed versus Damköhler number when the underlying one-dimensional flame is defined by a simple svmmetric counterflow configuration ( $1: 1$ stoichiometry, both Lewis numbers equal to 0.3 ), and negative speeds are not obtained. However, this might not be a commonly realizable phenomenon. In examining edge-flames constructed from the $S$ shaped response of the Kirkby-Schmitz configuration [12] (flux conditions for the fuel applied to one boundarv, Dirichlet conditions for the oxidizer applied at the other), for a variety of parameter choices, we have failed to identify robust edges even for Lewis numbers as small as 0.2 .

Clearly, the results presented here and in Ref. [8] could have relevance to the behavior of turbulent premixed flames in the laminar flamelet regime, particularly where the response of turbulent flame speed to turbulent intensity "bends."

\section{Conclusions}

inc:

Aif paper, we have examined two examples of mes (but note the remark following equation tho can arise when the reactants are premixed. foults for the symmetric (twin-flame) counter"ifiguration are similar to those of the singlefodifiguration examined in Ref. [8]. These can inmarized by the observation that if a hole is a juch a flame, it will get larger or smaller, ing on the value of the straining rate. This is (ing

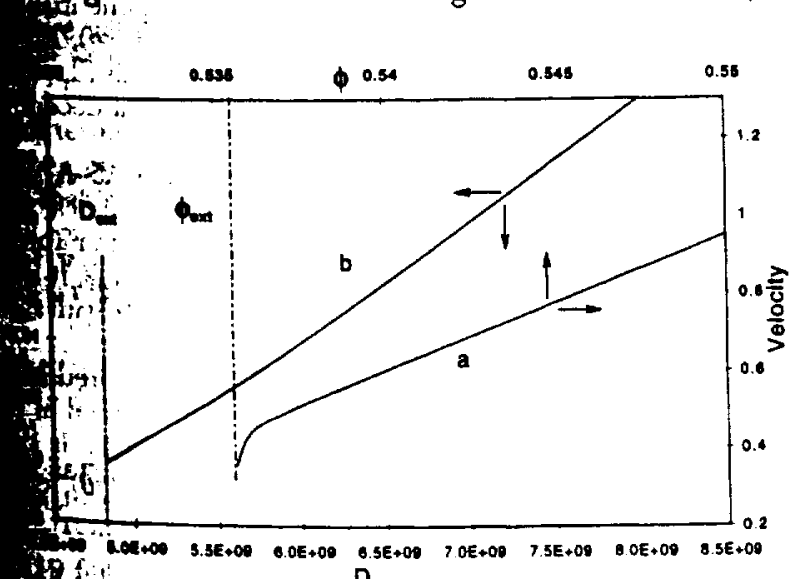

\section{Appendix \\ Asymptotic Solution of the One-Dimensional Counterflow Problem}

Here we briefly discuss the svstem of equations 4 and 5 in the asymptotic limit $\dot{\theta} \rightarrow \infty$; there are ingredients that have not been discussed before. In the limit, reaction is confined to a flame sheet located at $x=x_{*}$, and if $x_{*} \neq 0$, we have, to first order, 


$$
\begin{gathered}
\underline{0 \leq x<x_{*}} T=1+T_{f}=T_{t}, \quad Y=0 \\
\underline{x>x_{*}} T-T_{f}=1-Y / Y_{f} \\
=\operatorname{erfc}(x / \sqrt{2}) / \operatorname{erfc}\left(x_{*} / \sqrt{2}\right)
\end{gathered}
$$

The usual flame-sheet analysis determines the gradient on the unburned side of the flame,

$$
\begin{gathered}
Y_{f}^{-1} Y_{s}\left(x_{*}+0\right)=\left[2 D T_{a}^{4} \theta^{-2} e^{-m T_{n}}\right]^{1 / 2} \\
=\left\{S\left[\rho C_{p} / \alpha \lambda\right]^{1 / 2}\right\}_{*}=\bar{S}
\end{gathered}
$$

where $S$ is the adiabatic flame speed defined by the system. (The label ()$_{+}$is used to denote a quantity that is expressed in terms of dimensional variables.) Matching with the gradient of equation $A 1(b)$ then yields a formula for $x_{*}$,

$$
\left.\sqrt{2 / \pi} e^{-x \div / 2 / \operatorname{erfc}(x} / \sqrt{2}\right)=\xi
$$

and from this, we can deduce variations of $\tilde{x}_{*}$ with $\xi$, where

$$
\bar{x}_{*}=\left\langle S p C_{p} \dot{\lambda}^{-1} x_{*}\right\}_{+}=x_{*} S
$$

is a nondimensional flame-sheet location that is not scaled with $\alpha . \tilde{x}_{*}$ decreases monotonically with increasing $\alpha$, reaching the value 0 when $S^{-2}(\sim \alpha)=$ $\pi / 2$, a well-known result (e.g., Ref. [13]). For these solutions, the maximum temperature remains fixed at $T_{a}=1+T_{f}$.

There is also a solution for which $x_{*}=0$ and the flame temperature $T_{*}$ is within $O(1 / \theta)$ of $T_{a}$. Then to first order,

$$
\underline{x \geq 0} T=T_{f}+\operatorname{erfc}(x / \sqrt{2})
$$

Within the reaction zone, the variables are $t, \xi$ where

$$
T=T_{a}\left(1+\theta^{-1} T_{a} t\right), \quad x=\theta^{-1} \xi
$$

whence

$$
\begin{gathered}
t_{s t}=D \theta^{-2} e^{-w T_{a} t e^{t}} \\
t=t_{*}, \quad t_{s}=0 \text { at } \xi=0
\end{gathered}
$$

Here $t_{*}$ defines the flame temperature, a quantity to be determined.

A single integration leads to

$$
\begin{aligned}
& \left(t_{*}\right)^{2}=2 D \theta^{-2} e^{-\theta} / T_{a}\left[p\left(t_{*}\right)-p(t)\right], \\
& p(t)=e^{t}(1-t)
\end{aligned}
$$

which imposes the requirement $t \leq t_{*}<0$, a range in which $p$ is an increasing function. Matching be. tween the gradients defined by equations $A 5$ and $A 8$ $(x \rightarrow 0, t \rightarrow-\infty)$ then leads to a formula for $t_{*}$, namely,

$$
p\left(t_{*}\right)=2 \pi^{-15-2}
$$

defined for $\tilde{S}^{-1}<\pi / 2$. This branch. with infinite slope at $\tilde{S}^{-2}=\pi / 2$, is drawn in Fig. 1 (curve El) along with the upper branch $T_{*}=T_{a}\left(t_{*}=0\right)$ identified earlier when $x_{*} \neq 0$. Thus the asymptotics predicts a turning point (quenching point) in the response. but only provides a first-order estimate of its location $\left(T_{*}=T_{a}, \tilde{S}^{-2}=\pi / 2\right)$, and not a very accurate one at that.

A refinement of this description can only come from a higher-order analysis. and in this connection, we rewrite equation 4 in the form

$$
\begin{gathered}
T_{s s}+(\pi / 2) D e^{x^{2}}\left(T_{a}-T\right) e^{-1 / T}=0, \\
s=\operatorname{erf}(x / \sqrt{2})
\end{gathered}
$$

Now the turning point can only be generated when at least the exponential tail of the temperature perturbation within the reaction zone interacts with the symmetry plane $x=0$. The natural assumption is that $x_{*}=O(\ln \theta / \theta)$ when this occurs, corresponding to $e^{-k_{*}}=O(1 / \theta)$ for some $k$. Then we can approximate $e^{r^{2}}$ by 1 , and that this is a poor approximation beyond the reaction zone is obviously of no concern. Equation AlO can then be integrated using the boundary conditions

$$
T_{s}=0, \quad T=T_{*} \text { at } s=0
$$

to yield

$$
\begin{aligned}
\int_{T_{*}}^{T} d T\left[h\left(-\theta / T_{*}\right)-h(-\theta / T)\right]^{-1 / 2} \\
=-\sqrt{\pi \theta D} \cdot s \\
h(t) \equiv \\
\quad \frac{1}{2} \theta\left[E_{i}(t)-t^{-1} e^{t}-t^{-2} e^{t}\right] \\
+T_{a}\left[E_{i}(t)-t^{-1} e^{t}\right]
\end{aligned}
$$

where $E_{i}(x)=\int_{-\infty}^{x} d t t^{-1} e^{t}$ is the exponential integral. Equation A12(a), with $s=1(x=\infty), T=T_{f}$. is an implicit formula for $T_{*}(D)$, and this function is also plotted in Fig. 1 (curve A). It is a decent approximation to the exact solution.

If the problem implicit in Equations A5-A7 is examined to the next order, in an attempt to calculate a better approximation to the flame-temperature for the wall flame, that is, $t_{*}=t_{* 1}+\theta^{-1} t_{* 2}$, it is not difficult to show that $t_{* 2} \rightarrow \infty$ as $t_{* 1} \rightarrow 0$, with $t_{* 2} t_{* 1}$ $\rightarrow$ const., give or take logarithmic terms. (Secondorder flame-sheet theory is seldom required in our subject. but a detailed example is presented in Ref. [14].) Thus the erstwhile $1 / \theta^{2}$ term in $T_{*}$ is comparable to the $1 / \theta$ term if $t_{* 1}=O(1 / \sqrt{\theta})$, corresponding to an $O\left(1 / \theta_{\sqrt{ }} \bar{\theta}\right)$ deviation from $T_{n}$. The turning point is characterized by this scale. 
Consider the simplified (autonomous) version of Equation A10. with the variables of equation $A 6$. Then if the exponential is expanded.

ith infinite icurve El $=0$ ) idenptotics prein the rerimate of its $t$ a verv ac-

only come connection.

(),

rated when rature percts with the iumption is responding an approxroximation no concern. I using the

(Al1)

lential inte$\infty), T=T_{f}$ function is decent ap-

15-A7 is exto calculate perature for \#2, it is not with $t_{* 2} t_{* 1}$ 15. (Secondired in our ted in Ref. $T_{*}$ is com$\theta$, correin $T_{a}$. The Ue.

$$
d^{2} t / d \xi^{2}=\tilde{D}\left[t e^{t}-T_{0} \theta^{-1} t^{3} e^{t}+\ldots\right]
$$$$
\tilde{D} \equiv(\pi / 2) D \theta^{-2}-e^{-w / T_{n}}
$$

$$
g(t)=-\int_{-\infty}^{t} d t\left[t e^{t}-T_{n} \theta^{-1} t^{3} e^{t}+\ldots\right]
$$

(then $p(t)$ is the first approximation), the solution of equation $\mathrm{A} 13$ satisfying the boundary conditions equation $A 7(b)$ is

$$
\int d t\left[q\left(t_{*}\right)-q(t)\right]^{-1 / 2}=-\sqrt{2 \tilde{D}} \cdot \xi
$$

with large $-\xi$ behavior

$$
\begin{aligned}
& t \sim-\sqrt{2 \tilde{D} q\left(t_{*}\right)} \dot{\zeta}+t_{*}+\int_{-\infty}^{t} d t \times \\
& \left\{\left[1-q(t) / q\left(t_{*}\right)\right]^{-1 / 2}-1\right\}+\text { e.s.t. }
\end{aligned}
$$

Beyond the reaction zone,

and?

intur

$$
T_{\mathrm{ss}}=0
$$

with solution

tent

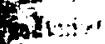

$$
T=A(\theta) s+T_{f}-A(\theta)
$$

-45 satisfy the boundary condition at $s=1$, where $\mathcal{L}(\theta)$ is a constant.

1. We now equate $T$ as defined by $A 16$ with $A 18$ 3itching to all orders) so that $A=-T_{a}^{2} \sqrt{2 \bar{D} q\left(t_{*}\right)}$ 3 ind

Tiver

$\lim \sqrt{2 D_{q\left(t_{n}\right)}}=\left(T_{n}-T_{f}\right)+T_{a}^{2} \theta^{-1} \times$

Tai :

$$
\left.\int_{-\infty}^{t} d t\left(\left[1-q(t) / q\left(t_{*}\right)\right]^{-1 / 2}-1\right\}\right]
$$

formula that describes variations of the flame temThere with the Damköhler number. Within the onitert of the autonomous problem, equation A19 Trilid to all algebraic orders since an arbitrary numGor of terms can be retained in $q$.

7if When only the first term in $q(t)$ is retained [the fim that is nominally $O(1)]$, the error in $t$ is $O(1) \theta)$, insll compared to $V_{\sqrt{\theta}}$, and the turning point is capinred, Fig. 1 (curve A1). Retaining two terms in $q(t)$ tves a better approximation, also shown in Fig. 1 (curve A2).

74. The term proportional to $\theta^{-1}$ in $A 19$ plays a crucial in defining the turning point: for at all orders, $f(0)=0$ so that the integral is loganithmically sinPular as $t_{*} \rightarrow 0$. (That all terms in the expansion of (equation Al4) have a zero derivative at the origin Teans that the expansion of each $q$-dependent term $\Delta 19$ for large $\theta$ is uniformly valid in $t_{*}$, further justification for the validity of replacing $q$ by its first approximation.) Indeed. for small $t_{*}$ and only the first approximation for $q, A 19$ can be approximated by

$$
\begin{gathered}
T_{a}^{2} \sqrt{2 \tilde{D} q\left(t_{*}\right)} \sim\left(T_{a}-T_{f}\right)+T_{a}^{2} \theta^{-1} \times \\
{\left[-\sqrt{2} \ln \left(-t_{*}\right)+1.34992 \ldots\right]}
\end{gathered}
$$

see Fig. 1 (curve B). The derivative of A20 is approximately

$$
\begin{aligned}
& (-1 / 2) \sqrt{2 \bar{D} t} t_{*}+(1 / \sqrt{2 \tilde{D}}) d \tilde{D} / d t_{*} \\
& \sim-\sqrt{2} /\left(\theta t_{*}\right)
\end{aligned}
$$

which explicitly shows that in the immediate neighborhood of the tuming point $t_{*}$ is $O(1 / \sqrt{\theta})$.

$A$ final note. The formula $A 16$ mav be used to provide a measure of the overall thickness of the reaction zone (bounded on the left by the svmmetry plane). The displacement effect can be calculated by finding the value of $\xi$ for which the linear variation of $t$, extrapolated to small $\xi$, defines a value of $t$ equal to $t_{*}$. Thus,

$$
\begin{aligned}
\xi_{*} & \sim T_{a}^{2}\left(T_{a}-T_{f}\right)^{-1} \times \\
& {\left[-\sqrt{2} \ln \left(-t_{*}\right)+1.34992 \ldots\right] }
\end{aligned}
$$

which is $O(\ln \theta)$ when $t_{*}$ is $O(1 / \sqrt{\theta})$. Recall the remarks following equation $\mathrm{A} 10$.

\section{Acknowledgments}

This work was supported by AFOSR and by the NASALewis Research Center. All of the problems described here were discussed, albeit incompletely, at the 16th Icders in Krakow, and the present report benefited from a number of conversations that two of us (GV, JB) enjoyed at that forum. Rather than offend by omitting some names through faulty recollection, we will simply acknowledge the constructive scientific climate that prevailed there. particularly in the Iwomic sessions.

\section{REFERENCES}

1. Kioni P. N., Rogg, B., and Bray, K. N. C., Combust. Flame 95:276-290 (1993).

2. Lee, S. T., Price, E., and Sigman. H.. J. Propul. Power 10:761-768 (1995).

3. Ross, H., Sotos, R., and T'ien, J. S., Combust. Sci. Technol. 75:155-160 (1991).

4. Buckmaster, J., J. Eng. Math. 31:269-284 (1997).

5. Müller, C. M., Breitbach, $H_{\text {., }}$ and Peters. N., in Twenty-Fifth Symposium (International) on Combus- 
tion, The Combustion Institute, Pittsburgh, 1994. pp. 1099-1106.

6. Dold, J. W., Combust. Flame 76:71-88 (1989).

7. Dold, J. W., Hartley, L. J., and Green. D., in Dynamical lssues in Combustion Theory (P. C. Fife, A. Liñán. and F. A. Williams, eds.) IMA Volumes in Mathematics and Its Applications, Springer, New York, 1991, pp. 83-105.

8. Vedarajan, T. G. and Buckmaster, J., Combust. Flame 114:267-273 (1998).

9. Jian-Bang, L. and Ronney, P. D., unpublished typescript.

10. Jarosinske. J., Strehlow, R. A., and Azarbarzin, A., in Nineteenth Symposium (International) on Combus- tion, The Combustion Institute. Pittsburgh, 1982, pp. 1549-1557.

11. Vedarajan. T. G., "Edge-flames in Premixed Combustion." Ph.D. thesis. University of Illinois. Urbana. January 1998.

12. Kirkby, L. L. and Schmitz, R. A., Combust. Flame 10:205-220 (1966).

13. Buckmaster, J. and Ludford. G. S. S., Lectures on Mathematical Combustion, SIAM, Philadelphia, 1983, p. 40.

14. Buckmaster, J. and Ronney, P. in Tuenty-Seventh Symposium (International) on Combustion. The Combustion Institute, Pittsburgh, 1998, 2603-2610.

\section{COMMENTS}

J. Chomiak, Chalmers University of Technology, Sweden. Your edge-flames are often curved. I wonder what is the mechanism of the branching phenomenon and how the branching affects the speed of the flames?

Author's Reply. The fresh/fresh counterflow edge-flame is horseshoe shaped, which is what one would expect. As for the edge-flame constructed from a deflagration with heat losses, it has only two choices: to be straight or curved, and in a two-dimensional asymmetric combustion field, it would be surprising if it were straight. The same can be said for our previous example of a premixed edge-flame, the one generated in a fresh/inert counterflow (Ref. [8] in the paper). The most obvious effect of edge curvature on the propagation speed occurs when the Lewis number (Le) is different from I, and we will report results for premixed flames in this case elsewhere. As we briefly note for diffusion edge-flames, small values of Le lead to enhanced reaction near the edge, and the edge-speed remains positive all the way to the $1-D$ quenching point. Indeed, edge advancement is possible beyond the 1-D quenching point: the edge trails cellular structures, sublimit flames-obviousiy, it can not trail a 1-D flame. This can also happen for the deflagration-with-heat-losses problem, even if $\mathrm{Le}=1$, for reasons that we discuss elsewhere.
Robert Pitz, Vanderbilt University, USA. Do you expect substantial differences in your conclusions if more complex chemistry is employed in your analysis?

Author's Reply. No. The essential characteristics of unbounded edge-flames-well defined waves that can travel with positive or negative speeds, depending on the $\mathrm{Da}$ mkohler number-have their roots in the fact that the underlying 1-D problem has two stable solutions. Whether the 1-D configuration is a diffusion flame or a premixed flame is a mere detail, as is the chemistry. Premixed failure waves (edge-flames with negative speeds) have, we believe, been seen in the sublimit failure of upward propagating methane-air flames in a standard inflammability tube, as we note in (Ref. [8] in the paper). Ronney has recently been able to establish stationary premixed edge-flames in his laboratory, and his observations are consistent with theory. These are analog-computer simulations with full chemistry. We can safely say that there are three, not two, basic flames in combustion: diffusion flames, deflagrations, and edge-flames. 